\title{
Solar prominences
}

\section{Brigitte Schmieder, Guillaume Aulanier and Tibor Török}

LESIA, Observatoire de Paris, 5 Place Janssen, Meudon, 92195, France

email: brigitte.schmieder@obspm.fr

\begin{abstract}
Solar filaments (or prominences) are magnetic structures in the corona. They can be represented by twisted flux ropes in a bipolar magnetic environment. In such models, the dipped field lines of the flux rope carry the filament material and parasitic polarities in the filament channel are responsible for the existence of the lateral feet of prominences.

Very simple laws do exist for the chirality of filaments, the so-called "filament chirality rules": commonly dextral/sinistral filaments corresponding to left- (resp. right) hand magnetic twists are in the North/South hemisphere. Combining these rules with 3D weakly twisted flux tube models, the sign of the magnetic helicity in several filaments were identified. These rules were also applied to the $180^{\circ}$ disambiguation of the direction of the photospheric transverse magnetic field around filaments using THEMIS vector magnetograph data (López Ariste et al. 2006). Consequently, an unprecedented evidence of horizontal magnetic support in filament feet has been observed, as predicted by former magnetostatic and recent MHD models.

The second part of this review concerns the role of emerging flux in the vicinity of filament channels. It has been suggested that magnetic reconnection between the emerging flux and the pre-existing coronal field can trigger filament eruptions and CMEs. For a particular event, observed with Hinode/XRT, we observe signatures of such a reconnection, but no eruption of the filament. We present a 3D numerical simulation of emerging flux in the vicinity of a flux rope which was performed to reproduce this event and we briefly discuss, based on the simulation results, why the filament did not erupt.
\end{abstract}

Keywords. Solar prominence, magnetic emerging flux, eruption, 3D MHD model

\section{Introduction}

Solar filaments (or prominences) are cool and dense plasma embedded in the hot corona. The global structure of filaments can sometimes be observed over several solar rotations. Their pressure-scale height is less than $500 \mathrm{~km}$ and does not allow static plasma to remain in prominences as high as $10-50 \mathrm{Mm}$. The plasma in filament fine structures is dynamic (Schmieder et al. 1991, Zirker et al. 1998, Lin et al. 2005). Recent observations of Hinode give the impression of continuous downflows in vertical structures (Figure 1a). However, the velocities do not exceed $\approx 10 \mathrm{kms}^{-1}$, unless the filament is particularly activated. The role of the dynamics is still unclear. Therefore it is reasonable to assume that filaments are magnetically supported in the corona, the plasma being frozen in magnetic structures. Magnetic flux tubes and arcades have been proposed in theoretical models to support the filament material (e.g. Amari et al. 1999, Antiochos et al. 1994). Extrapolations of photospheric magnetic fields show that filaments can be indeed modelled by flux tubes with plasma filling the dips. Since then, this "magnetic dip filling" procedure has been applied by various groups to perform model predictions (Aulanier et al. 2000) and to analyze real observations with linear magnetohydrostatic, non-linear magnetofrictional and fully MHD models (Aulanier \& Schmieder 2002, Lionello et al. 2002, van Ballegooijen 2004, Bobra et al. 2008). These topologies have also recently been found to be consistent with the evolution of the photospheric vector magnetic field during a filament formation resulting from flux emergence, as observed by 


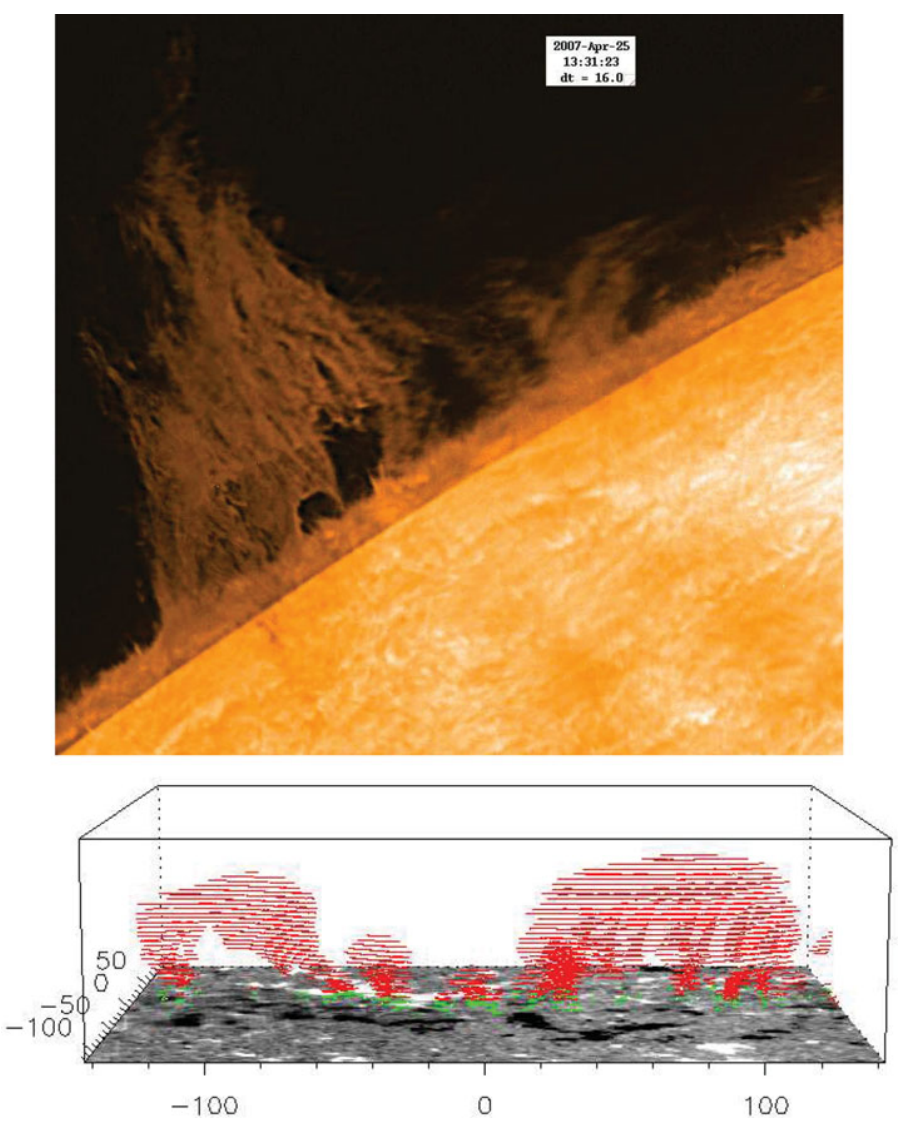

Figure 1. top panel: Hinode prominence observed on 25 April 2007, low panel, Prominence modeling by the dips of field lines extrapolated from an observed photospheric magnetogram of THEMIS (Dudik et al. 2008).

Hinode/SOT (Okamoto et al. 2008). The barbs and footpoints are mimicked by the dips of field lines which deviate from the main flux tube and are rooted in parasitic polarities, minor polarities of opposite sign as their environment.

Due to their large scales and multi-wavelength manifestations, solar filaments and prominences are key phenomena for the study of high-stressed non-potential currentcarrying magnetic fields in the solar corona. They can be used to understand how magnetic helicity slowly accumulates in the Sun's corona, and is then later ejected in the heliosphere in the form of coronal mass ejections, which are known to be the main drivers of extreme space weather. In spite of impressive progress with the Advanced Stokes Polarimeter (ASP) for the 2D measurement of the internal magnetic field in prominences (Casini et al. 2003), building a 3D picture still requires the combination of multi-wavelength observations and magnetic models (e.g. Dudik et al. 2008), see (Fig. 1b).

In this context, observational laws have been put forward from the chirality of observed features (e.g. chromospheric fibrils, lateral filament feet, overlaying coronal arcades) to derive the direction of the axial magnetic field inside solar filaments. These are the socalled "filament chirality rules". They state that a dominant fraction of filaments located in the northern (resp. southern) solar hemisphere have right- (resp. left-) bearing feet and fibrils, left (resp. right) skewed arcades, and dextral (resp. sinistral) internal axial fields, which point rightward (resp. leftward) as the filament is viewed from the main 
positive polarity field on the side of the photospheric inversion line above which it is located (Martin et al. 1994, Martin 1998).

When combined with the chirality rules, these models predict that dextral (resp. sinistral) filaments correspond to left- (resp. right) hand magnetic twists, hence to negative (resp. positive) magnetic helicities.

Here we report on the use of all these concepts and tools, firstly for the disambiguation between dipped and arcade topologies as observed with THEMIS below a filament foot, and secondly for quantifying the role of relative magnetic helicities between two filaments to predict either their subsequent merging into a longer and potentially less stable structure, or their interaction in the form of a confined flare.

In the second part of the paper we study the conditions to get filament eruption. Most filaments eventually erupt in many cases as part of a coronal mass ejection (CME). Such eruptions are often preceded by detectable changes in the photospheric magnetic field in the vicinity of the filament. Here we focus on emerging flux in the vicinity of filament channels. It has been suggested that magnetic reconnection between the emerging flux and the pre-existing coronal field can trigger filament eruptions and CMEs. For a particular event, observed with Hinode/XRT, we observe signatures of such reconnection, but no eruption of the filament. We present a numerical simulation of this event and we briefly argue why no eruption took place in this case.

\section{Role of the magnetic helicity}

\subsection{Resolution of the 180 degree of ambiguity and observations of magnetic dips}

A debate is raging in the solar physics community about the magnetic nature of filament feet, which are common underlaying and lateral extensions observed in absorption on the solar disc in $\mathrm{H} \alpha$ and in the EUV. These feet connect filament bodies suspended in the corona to the lower atmospheric layers. Are these feet formed by continuously injected plasma condensation in magnetic arcades, as hinted by some observations and conceptual models (Martin 1998), or do they consist of quasi-static condensations that are maintained against free-fall by the Lorentz force in a low-lying continuous distribution of magnetic hammocks, from the feet ends to up to the filament bodies, as first predicted by linear force-free field and magnetohydrostatic models (Aulanier \& Démoulin 1998, Aulanier \& Schmieder 2002, Dudik et al. 2008)? This debate had been lacking of new discriminators for about ten years, until this issue was recently addressed through new direct measurements of the photospheric magnetic field vector $\vec{B}$ in a filament channel located far from the center of the solar disc, resulting from the PCA-based inversion of high-precision spectropolarimetric observations with the MTR instrument of the THEMIS telescope (López Ariste et al. 2006).

A major problem with these measurements is that they still give the direction of the component of the magnetic field vector on the plane of the sky at $\pm 180^{\circ}$. This fundamental ambiguity does not allow the observations, taken alone, to state whether an arcade or a dip is measured at a given place. So as to solve this paradigm, chirality rules can be applied to the disambiguation of the measured transverse magnetic fields, before deprojecting them to obtain the three components of the magnetic field vector in the reference frame of the solar surface. This procedure was proposed and applied in López Ariste et al. (2006), and rephrased by Martin et al. (2008). The studied filament was identified to be sinistral, hence with a magnetic field vector globally pointing toward the left, as viewed from the dominant positive magnetic polarity in the photosphere (Fig. 1, top). Interestingly, it was found that, for almost every area analyzed in detail within the 
observed filament channel, only the sinistral solution that matched the chirality rule on the plane of the sky remained sinistral in the reference frame of the solar surface. Using the chirality-consistent solution to calculate the curvature $B^{2} /(\vec{B} \cdot \vec{\nabla}) \vec{B}$ of the magnetic field at various places within the channel, the first-ever $3 \mathrm{D}$ magnetic dip topology was found in the photosphere below a filament foot from observations (Fig. 2, bottom). This is consistent with early linear force-free models for filament feet (Aulanier \& Démoulin 1998) and with recently recovered in MHD simulations of prominence formation by twisted magnetic flux tube emergence through the photosphere (Magara 2007).

\section{2. $H \alpha / E U V$ observations and $M H D$ simulations of merging/flaring filament sections}

Even from previous papers it was mentioned that filaments can only merge if their chirality is the same (see e.g. Malherbe 1989, Martin et al. 1994, Rust 2001, van Ballegooijen 2004), this condition had never been tested with dedicated observational and theoretical studies until recently.

The recent multi-wavelength analysis of three interacting filament sections F1, F2 and F3 (Fig. 3) observed during several days during a Joint Observing Programme between ground-based instruments in the Canary Islands (the SVST and the MSDP on the VTT) space-borne satellites (here we only refer to TRACE images), was the first dedicated observational study of this issue. Following their evolution over several days, it was shown that F1 and F2 gently merged into a single structure, as observed by a gradual filling in $\mathrm{H} \alpha$ of the gap R1 between both of them. This merging was associated with mild EUV brightenings and with slow $\mathrm{H} \alpha$ Doppler flows at the merging point (Schmieder et al. 2004). While EUV brightenings are a good indicator of magnetic reconnection, the flows revealed that the merging first took place by dynamic exchanges between the two progenitors, until they formed a more stable single long quiet filament. Two days later F2 and F3 produced a confined flare, manifested by the formation of new long EUV post-flare loops, as they got into contact at the point R2 (Deng et al. 2002). In order to
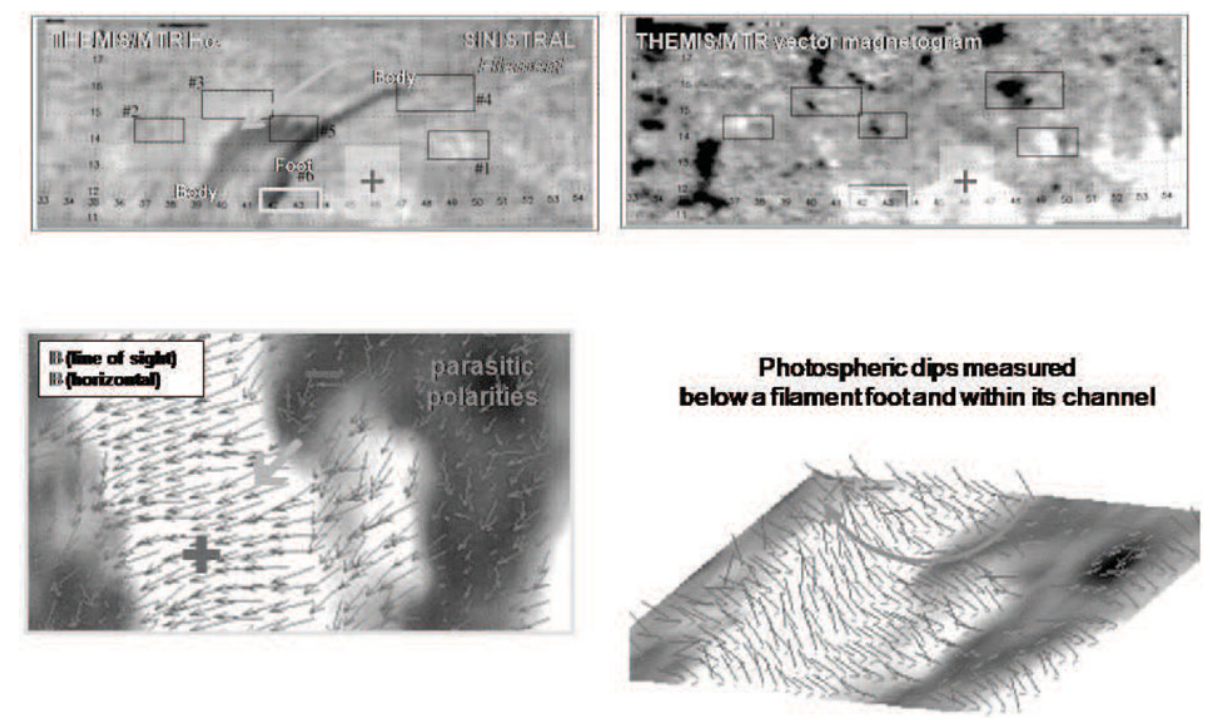

Figure 2. First-ever dentification of a magnetic dip at the footpoint of a filament foot. The vector magnetic field was measured with THEMIS/MTR and the $180^{\circ}$ ambiguity was solved using usually observed filament chirality rules. The transverse fields which have an inverse orientation from a - toward a + polarity indicate the presence of magnetic dips above the associated inversion line. (adapted from López Ariste et al. 2006). 

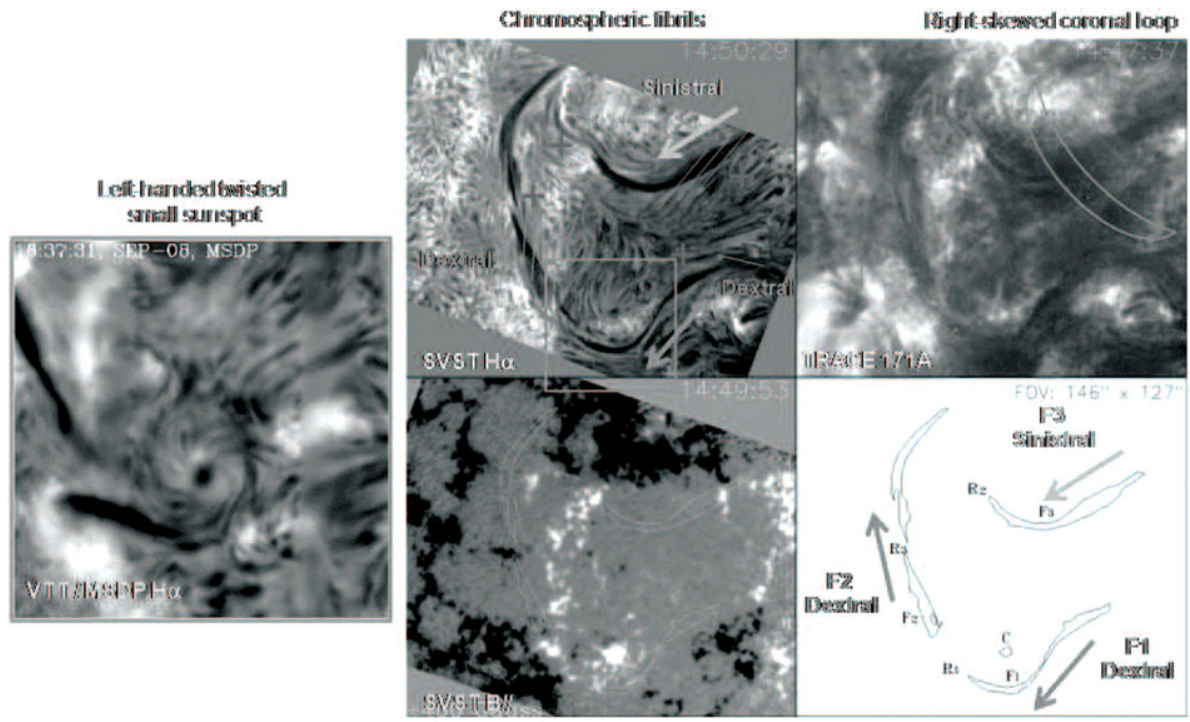

Figure 3. Identification of the chiralities of three interacting filaments $\mathrm{F} 1,2,3$, using various observed features. F1 and F2 were observed to merge in the R1 area, whereas a flare took place between F2 and F3 one day later, as they interacted but did not merge (adapted from Deng et al. 2002 and Schmieder et al. 2004).

address the role of helicity in these two events, Schmieder et al. (2004) used the chirality rules for chromospheric fibrils and magnetic field polarity, overlaying coronal arcades and handedness of neighboring sunspots, so as to predict the direction of the axial fields in the filaments. The resulting axial fields are indicated by arrows in the upper-middle panel of Fig. 3. The results of this study were numerous. It was confirmed that when two filaments interact, magnetic reconnection takes place and leads to a sudden flare (resp. a gradual merging) when their helicity signs are of the opposite (resp. the same) sign. It was also shown that magnetic helicity must slowly accumulate prior to filament merging, as seen by the rotation of a small twisted sunspot in the vicinity of the merging point. Finally, it could be deduced that in its early stages, magnetic reconnection accelerates plasma between the previously split filaments, and that it must later result in a change of topology which can sustain quiet and almost non-moving filament material all along the newly formed filament. All these put strong constraints on the MHD modeling of filament merging and flaring.

Numerical MHD simulations of the formation, interaction, and magnetic reconnection between pairs of solar filaments have then been conducted. Line-tied sub-Alfvénic shearing boundary motions were applied to adjacent and initially current-free magnetic bipoles. The simulations were performed in a low- $\beta$ adiabatic regime, using $500 \times 190 \times 190$ mesh points in a non-uniformed grid, with a Flux Corrected Transport scheme that allows reconnection owing to numerical diffusion at the scale of the mesh. Four possible combinations of chiralities (identical or opposite) and axial magnetic fields (aligned or opposed) between the participating filaments were considered (DeVore et al. 2005). It was found that, when the topology of the global flux system comprising the prominences and arcades is bipolar, so that a single polarity inversion line is shared by the two structures, then identical chiralities necessarily imply identical magnetic helicity signs and aligned axial fields. In this case, finite-B slipping magnetic reconnection formed new field lines linking the two initial prominences (see Fig. 4, left). At early times, shear Alfvén waves 

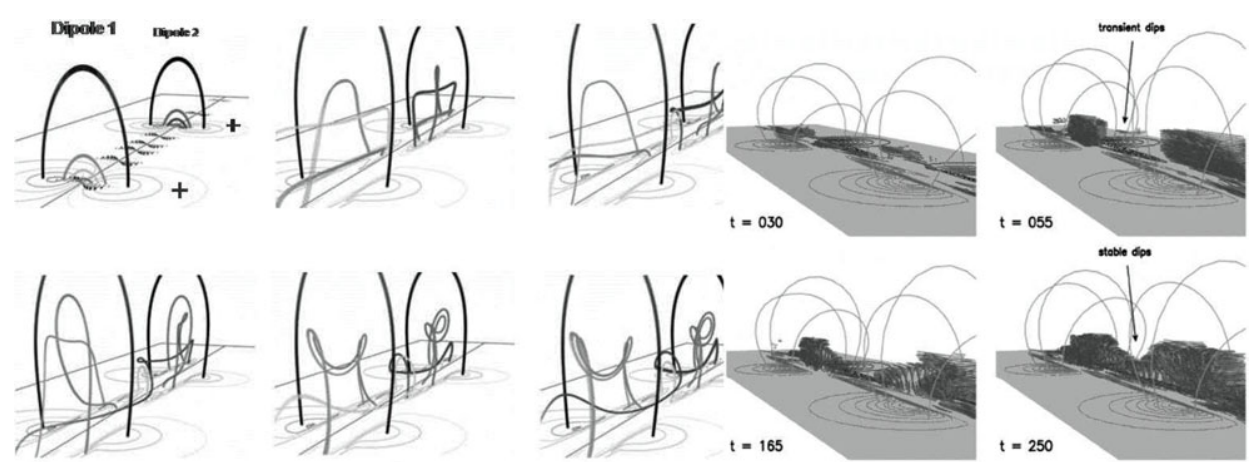

Figure 4. 3D MHD simulation of prominence merging, resulting from finite-B magnetic reconnection between two dipoles that share a common photospheric inversion line, and whose shear result in the same magnetic helicity sign. (Left:) Reconnecting field lines. (Right:) Resulting distribution of plasma-supporting magnetic dips, simulating the prominence material (adapted from DeVore et al. 2005 and Aulanier et al. 2006).

propagated through these newly reconnected field lines, which can accelerate plasma condensations from one progenitor to another. As the shear increases, a new distribution of magnetic dips formed and increasingly filled the volume between both progenitors, so that they gradually merged into a single filament. We identified the multistep mechanism, consisting of a complex coupling between photospheric shear, slipping magnetic reconnection in the corona, and formation of quasi bald patches, that is responsible for stable filament merging through dip creation (Aulanier et al. 2006). This first model successfully reproduced the observations of filament merging by Schmieder et al. (2004). The second model, which also made use of a large-scale bipolar field, but which induced opposite helicities and axial fields between the two prominences, hardly resulted in any magnetic reconnection. The resulting lack of merging is consistent with the observations of Deng et al. (2002), although no flare reconnection occured in the model. When the topology instead is quadrupolar, so that a second polarity inversion line crossing the first lies between the prominences, then the converse relation holds between chirality and axial-field alignment. Reconnections that form new linking field lines now occur between prominences with opposite chiralities. They also occur, but only result in footpoint exchanges, between prominences with identical chiralities. These findings do not conflict with the observational rules, since the latter have yet to be derived for non-bipolar filament interactions; they provide new predictions to be tested against future observational campaigns.

\section{Flux emergence}

The association between emerging flux and filament eruptions has been extensively studied by Feynman and Martin (1995). They found that in 17 out of 22 cases where newly emerging flux in the vicinity of filaments could be observed, the filament erupted, whereas in the remaining 5 cases it did not. The new flux typically started to emerge a few days before the eruption, indicating a slow evolution towards an unstable state before eruption. In 26 out of 31 cases where no emerging flux in the vicinity of the filament was detectable, the filament did not erupt within the period of observation. The authors concluded that filament eruptions are associated with newly emerging flux, but that the latter is not a necessary condition for eruption. In a more recent study, Jing et al. (2004) found that 54 out of 80 filament eruptions were associated with flux emergence. 


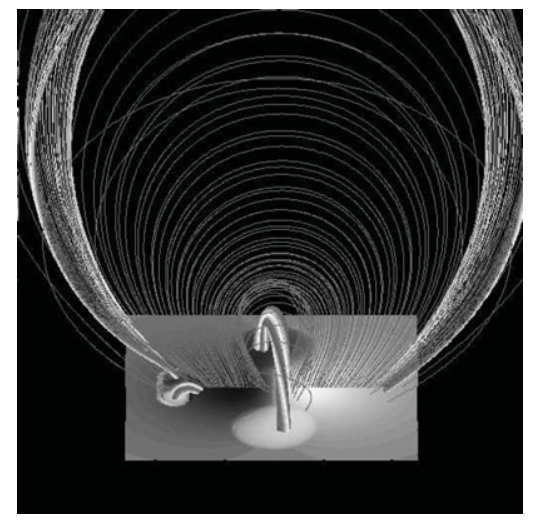

Figure 5. Snapshot from a 3D simulation of flux emergence in the vicinity of a coronal flux rope (from Török 2009). The TD flux rope is visible in the centre. To the left, a small flux rope is emerging and reconnecting with the potential field overlying the TD flux rope.

These results indicate that in about $60-70 \%$ of all cases, newly emerging flux in the vicinity of a filament leads to its eruption. But how can the new flux drive the filament towards eruption? Magnetic reconnection seems to be the key. It was suggested that the emerging flux reconnects with the field overlying the filament and hence destabilizes it (Feynman and Martin 1995) or decreases its tension to a degree that the core flux can not longer be stabilized and erupts (Wang et al. 1999). However, as pointed out in Feynman and Martin (1995) and demonstrated in 2.5D numerical simulations (Chen and Shibata 2000), the magnetic orientation of the emerging flux with respect to the preexisting coronal field must be "favorable" for reconnection to occur. In the next section, we present a recent observation of signatures of such reconnection.

Reconnection is not a necessary condition for eruptions. Eruptive prominences can result directly from instabilities (kink instability, torus instability) leading to loss of equilibrium in the relevant structures (Lin, Forbes and Isenberg 2001, Lin and Forbes 2000, Low 2001, Török and Kliem 2005). In principle disruption occurring in a magnetic structure results from the interaction between the current and the magnetic field in the relevant configuration.

\subsection{The event on 2007 April 24}

Figure 6 shows a recent example of flux emergence close to a filament channel observed by Hinode/XRT on 2007 April 24. The bright X-ray loops indicate that reconnection between the emerging flux and the magnetic field overlying the filament took place. Two main bright systems of loops are visible, one to the left of the filament channel and one arching above the filament, outlining the edge of the filament cavity. We also observed a brightening propagating along this arch in the early phase of the evolution. The filament is not destabilized by the reconnection and does not erupt in this case.

\subsection{D MHD simulation}

In order to understand the interaction of the emerging flux with the pre-existing coronal field, and to understand why the filament did not erupt, we aimed to reproduce this event in an MHD simulation. The latter will be described in detail elsewhere (Török et al. 2009), here we just give a brief summary of the setup and the results.

As initial condition for the simulation we use the analytical model of a bipolar active region by Titov \& Démoulin (TD) (1999). The model consists of a force-free, line-tied and twisted coronal flux rope embedded in a potential field arcade. Numerical simulations 

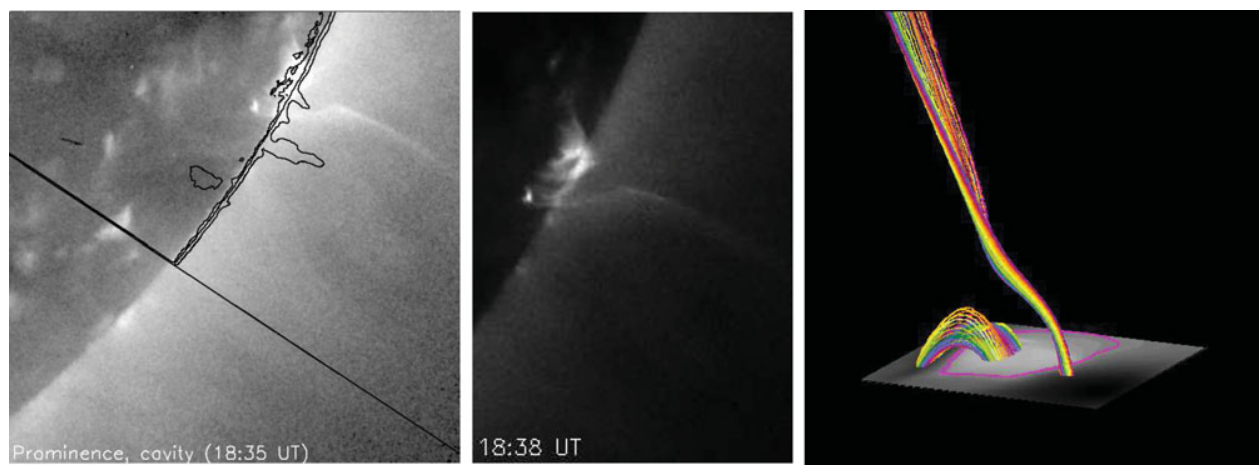

Figure 6. Left: Hinode/XRT image ( 512 x 512 arc sec) overlaid by contours of the $\mathrm{H}_{\alpha}$ prominence observed on 2007 April 25 at 13:38 UT by MSDP. A large cavity is seen above the prominence. Centre: The flaring X-ray loops. Right: The region of flux emergence in the numerical simulation described in the text. The TD flux rope is located to the right of the zoomed region. Selected newly reconnected magnetic field lines are shown. The field lines qualitatively resemble the shape of the two main X-ray loops visible in the central panel.

(Török et al. 2004, Török and Kliem 2007) have demonstrated that the TD flux rope can be subject to the helical kink instability and the torus instability (Kliem and Török 2006). The morphological and kinematic evolution of an erupting filament could be successfully reproduced in another simulation (Török and Kliem 2005, Schrijver et al. 2008). For our simulation, we choose the parameters of the TD model such that the flux rope is initially stable with respect to both instabilities.

We then mimic the emergence of new magnetic flux by successively changing the boundary conditions at the bottom plane of the numerical domain (the "photosphere") such that the slow and rigid emergence of another, smaller and uniformly twisted flux rope in the vicinity of the pre-existing rope is modeled (see Figure 5 for a simulation similar to the one described here; see also Fan and Gibson 2004).

As the new flux rope slowly emerges, a magnetic null point is gradually formed slightly above it, within the current sheet which forms at the interface between the two flux ropes, and the emerging rope starts to reconnect with the potential field arcade overlying the TD rope. The amount of flux reconnected depends on the strength and orientation of the magnetic field within the emerging rope with respect to the pre-existing coronal field. We choose the orientation of the rope's field such that it is favorable for reconnection. As the reconnection proceeds, new connectivities are formed (Figure 6, right): field lines rooted in the positive (white) polarity of the emerging flux rope now close down in neighboring regions of negative polarity (black) of the TD model and form low-lying arcades, whereas field lines starting from the negative polarity of the emerging rope reconnect with field lines initially overlying the TD rope. The latter exhibit a kinked shape, just as the bright X-ray loop overlying the filament. This kinked shape corresponds to the field geometry around the 3D null point, which has a typical fan surface and spine field line. Although our simulation does not treat the thermodynamics, it is legitimate to qualitatively compare our simulation with the XRT observations, since newly reconnected field lines are expected to be heated and to brighten in X-ray images. The shapes of the newly reconnected field lines are qualitatively similar to the shape of the bright Xray features, indicating that the simulation, and its resulting topology, reproduces the magnetic interaction between emerging and pre-existing flux reasonably well.

As the filament in reality, the TD flux rope does not erupt in our simulation. As mentioned earlier, observations indeed show that emerging flux in the vicinity of filaments 
does not necessarily lead to an eruption. The question arises under which circumstances flux emergence can trigger filament eruptions and CMEs. We think the main factor which decides on the occurrence of an eruption triggered by flux emergence in the vicinity of filaments is how "far" the pre-eruptive coronal configuration is from an unstable state at the time when the reconnection between the emerging flux and the arcade field above the core flux carrying the filament sets in. The reconnection must sufficiently weaken the tension of the overlying field for the core flux to erupt. How effective the reconnection is in this respect will depend on many factors, as for example the field orientation and the spatial distance of the emerging from the core flux. In cases where the core flux is already close to instability, a small amount of new flux emergence might be sufficient to trigger its eruption, whereas in other cases even a large amount of emerging flux might not be able to drive the system towards an unstable state. It seems that the event described above belongs to the latter category. We note that in cases where the new flux emerges just below the filament, it might reconnect directly with the core flux. This could increase the twist of the core flux such that it erupts even if the overlying filed is not weakened significantly. Similar conclusions have been drawn in earlier observational, analytical and numerical studies on the relation between emerging flux and solar eruptions. For a more detailed discussion, we refer the reader to the corresponding papers (e.g. Wang et al. 1999, Notoya et al 2007, Chen and Shibata 2000, Lin et al. 2001).

\section{Summary}

Making use of observational chirality rules and theoretical magnetic modeling for filament magnetic fields, we have obtained several important breakthroughs which can now be re-used in future studies. First, a chirality-based disambiguation technique in a THEMIS vector magnetogram of a filament channel have been applied (López Ariste et al. 2006); this led to the first observational evidence of magnetic support in filament feet. Second we have analyzed with unprecedented accuracy the interaction between three distinct filaments, for which we identified the sign of magnetic helicity in several independent ways; this led to the observational conjecture that filament mergers must exhibit the same sign of helicity, otherwise their interaction will result in a confined flare (Schmieder et al. 2006). Third a parametric study of sheared dipoles with 3D MHD calculations, varying their initial orientation and the direction of their associated shearing motions confirmed the observational conjecture, and extended its results to potential mergers with opposite helicity signs if their progenitor bipoles are oppositely oriented (Aulanier et al. 2006). Finally we have presented a new 3D simulation of emerging flux close to a coronal flux rope and discussed that both the "distance" of the flux rope from instability and the "effectivity" of reconnection in driving the rope towards instability will determine whether filament eruptions can be triggered by flux emerging in their vicinity.

\section{Acknowledgements}

Financial support by the European Commission through the SOLAIRE network (MTRM-CT-2006-035484) is gratefully acknowledged.

\section{References}

Amari, T., Luciani, J. F., Mikic, Z., \& Linker, J. 1999, ApJ, 518, L60

Antiochos, S. K., DeVore, C. R., \& Klimchuk, J. A. 1999, ApJ, 510, 495

Aulanier G. \& Démoulin, P. 1998, A\&A, 329, 1125 
Aulanier, G., Srivastava, N., \& Martin, S. F. 2000, ApJ, 543, 447

Aulanier, G. \& Schmieder, B. 2002, A\&A, 386, 1106

Aulanier, G., DeVore, C. R., \& Antiochos, S. K. 2006, ApJ, 646, 1349

Bobra, M., van Ballegooijen, A. A., \& DeLuca, E. E. 2008, ApJ, 672, 1209

Casini, R., López Ariste, A., Tomczyk, S., \& Lites, B. W. 2003, ApJ, 589, L67

Chen, P. F. \& Shibata, K. 2000, ApJ, 545, 524

Deng, Y., Schmieder, B., \& Engvold, O. 2002, Sol. Phys., 209, 153

DeVore, C. R., Antiochos, S. K., \& Aulanier, G. 2005, ApJ, 629, 1122

Dudik, J., Aulanier, G., Schmieder, B., Bommier, V., \& Roudier, T. 2008, Sol. Phys., 248, 29

Fan, Y. \& Gibson, S. E. 2004, ApJ, 609, 1123

Feynman, J. \& Martin, S. F. 1995, J. Geophys. Res., 100, 3355

Jing, J., Yurchyshyn, V. B., Yang, G., Xu, Y., \& Wang, H. 2004, ApJ, 614, 1054

Kliem, B. \& Török, T. 2006, Phys. Rev. Lett., 96, 255002

Lin, J. 2004, Solar Physics, 219, 169

Lin, J. \& Forbes, T. G. 2000, J. Geophys. Res., 105, 2375

Lin, J., Forbes, T. G., \& Isenberg, P. A. 2001, J. Geophys. Res., 106, 25053

Lin, Y. L., Wiik, J. E., \& Engvold, O. 2003, Solar Phys., 216, 109

Lin, Y. L., Wiik, J. E., Engvold, O., et al., 2005, Solar Phys., 227, 283

Low, B. C. 2001, J. Geophys. Res. 106, 25141

López Ariste, A., Aulanier, G., Schmieder, B., \& Sainz Dalda, A. 2006, A\&AA, 456, 725

Lionello, R., Mikic, Z., Linker, J., \& Amari, T. 2002, ApJ, 581, 718

Malherbe, J.-M. 1989, in Dynamics and Structure of Quiescent Solar Prominences, Kluwer Ac. Pub., 115

Magara, T. 2007, PASJ, 59, L51

Martin, S. F., Bilimoria, N., \& Tracadas, P. W. 1994, in Solar Surface Magnetism, Kluwer Ac. Pub., 303

Martin, S. F. 1998, Sol. Phys., 182, 107

Martin, S. F., Lin, Y., \& Engvlod, O. 2008, Sol. Phys., 250, 31

Notoya, S. et al., 2007, ASP Conf. Series, 369, 381

Okamoto, T. J., Tsuneta, S., Lites, B., et al., 2008, ApJ, 673, L215

Rust, D. M. 2001, in Encyclopedia of Astronomy and Astrophysics, http://eaa.iop.org

Schmieder, B., Mein, N., Deng, Y., et al., 2004, Sol. Phys., 223, 119

Schrijver, C. J., Elmore, C., Kliem, B., Török, T., \& Title, A. M. 2008, ApJ, 674, 586

Titov, V. S. \& Démoulin, P. 1999, A\&A A, 351, 707

Török, T. 2009, in preparation

Török, T., Kliem, B., \& Titov, V. S. 2004, A\&SA, 406, 1043

Török, T. \& Kliem, B. 2007, Astronomische Nachrichten, 328, 743

Török, T., Schmieder, B., \& Aulanier, G. 2009, in preparation

van Ballegooijen, A. A. 2004, ApJ, 615, 519

\section{Discussion}

Nindos: I have observed a couple of cases where close to a filament, reconnection-favored bipoles emerge. But the filaments didn't erupt. Just for your information.

SCHMIDER: Thank you for your remark. With simulation we can study the conditions of eruptions using a large space of parameters. 